\title{
EDITORIAL
}

\section{A stronger role for science}

\author{
Drug development is inherently risky, but if perceived short-term business imperatives \\ outweigh sound science in decision-making, the magnitude of these risks might become \\ unsustainable.
}

In our March issue last year, we featured an extensive analysis ${ }^{1}$ of the research and development $(\mathrm{R} \& \mathrm{D})$ productivity challenges that underlie the ongoing restructuring by the pharmaceutical industry. As well as identifying the relative contributions of the various steps in the drug discovery process to overall R\&D productivity, the article also highlighted possible causes of the hugely costly failures in late-stage clinical development. In this respect, the authors observed that "we consider that one of the major contributors to high Phase III attrition is simply premature advancement of NMEs [new molecular entities] into Phase III", also noting that this "is often the result of poor discipline in portfolio management combined with perceived near-term business imperatives."

Some insights into the potential influence of such factors in one of the most prominent Phase III failures in recent years - the termination of the development of Pfizer's cholesteryl ester transfer protein (CETP) inhibitor torcetrapib in December 2006, following $\sim$ US $\$ 800$ million of R\&D investment - are provided in a news feature on page 163. Although our understanding of the possible reasons for the dramatic failure of torcetrapib has improved since 2006 (and has been important in the decisions of Merck and Roche to continue development of their CETP inhibitors), signs of one key risk of torcetrapib were apparent before it failed: a modest increase in blood pressure was observed early during development. When Phase III trials were initiated, experts believed that this undesirable effect for a drug intended to reduce cardiovascular disease risk would be outweighed by the substantial positive effects of torcetrapib on the levels of 'good' high-density lipoprotein cholesterol. However, it now seems that the rise in blood pressure with torcetrapib was linked to an increase in aldosterone levels, which has well-known detrimental effects in patients with cardiovascular disease.

Given this early indicator of a risk and the eventual outcome, the obvious question is why the development of torcetrapib was pursued, rather than that of follow-up compounds that did not increase blood pressure. In this case, it seems (see page 163) that commercial pressures to replace the vast loss of revenue resulting from the imminent expiration of patent protection for Pfizer's atorvastatin (Lipitor) — which has had annual sales exceeding
$\$ 10$ billion - were a major influence in the decision to progress with torcetrapib, rather than delay and select a follow-up compound. So, with the benefit of hindsight, the case of torcetrapib could provide a strong example of the substantially increased risk of failure when commercial influences outweigh science in R\&D decision-making.

It could also be illuminating to understand the extent to which such influences might have played a role in more recent Phase III failures, such as those of several oncology compounds (see page 165). Interestingly, a recent analysis ${ }^{2}$ reported that the current failure rate at the Phase III or regulatory submission stage is $\sim 50 \%$, and of the 83 failures at this stage between 2007 and 2010, 28\% occurred in the field of oncology. Given that oncology has been one of the most active areas of $\mathrm{R} \& \mathrm{D}$ in the past few years, this is perhaps not surprising. However, questions are increasingly being asked about a range of issues related to oncology trials, such as whether the designs, even if successful, are well suited to demonstrate genuinely meaningful benefits ${ }^{3}$. Of even greater concern is the possibility that for some companies - particularly those whose value is entirely based on products in development - commercial incentives could encourage poor decisions to pursue the development of compounds that only generate weak evidence of effectiveness at Phase II and have an even higher risk than typical of failure at Phase III ${ }^{4}$. Such decisions not only ill serve the patients involved in the particular trials and the investors in the company, but also harm oncology drug development and the long-term willingness for investment in pharmaceutical R\&D in general.

Overall, given the increasing appreciation of the complexity of human disease and the associated uncertainty about how best to intervene therapeutically in any given setting, pharmaceutical R\&D will remain a risky endeavour. If it is also to remain a sound investment, the evidence is growing that sound science must play a stronger role in the decisions to take these risks.

\footnotetext{
1. Paul, S. M. et al. How to improve R\&D productivity: the pharmaceutical industry's grand challenge. Nature Rev. Drug Discov. 9, 203-214 (2010).

2. Arrowsmith, J. Phase III and submission failures: 2007-2010. Nature Rev. Drug Discov. 10, 87 (2011)

3. Ocana, A. \& Tannock, I. F. When are "positive" clinical trials in oncology truly positive? J. Natl Cancer Inst. 103, 16-20 (2011)

4. Fiorino, T. Industry, clinical trials and the cost of cancer drugs: an investor's perspective. J. Clin. Oncol. 25, e21-e23 (2007).
} 